\title{
USING THE RADIATION OF HYDROGEN ATOMS AND MOLECULES TO DETERMINE ELECTRON DENSITY AND TEMPERATURE IN THE LINEAR PLASMA DEVICE PSI-2
}

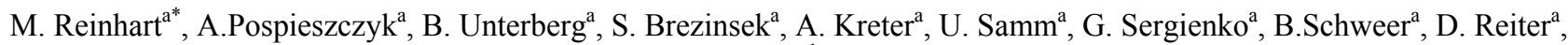 \\ D. Wünderlich ${ }^{\mathrm{b}}, \mathrm{U}$. Fantz $^{\mathrm{c}}$ \\ a) Institute of Energy- and Climate Research - Plasma Physics, Forschungszentrum Jülich GmbH, Association \\ EURATOM - FZ Juelich, Partner in the Trilateral Euregio Cluster, D-52425 Jülich, Germany \\ b) Max-Planck-Institute of Plasma Physics, EURATOM Association, Boltzmannstr. 2, D-85748 Garching, Germany \\ c) Chair of Experimental Plasma Physics, Augsburg University, Universitätsstr. 1, D-86135 Augsburg, Germany \\ *m.reinhart@fz-juelich.de
}

In this work we investigate the applicability of several optical emission spectroscopy methods to measure the electron density and temperature in deuterium plasma in the linear plasma generator PSI-2. The spectroscopy measurements are realized by an imaging spectrometer which delivers radial profiles of the emission lines. With the application of an inverse Abel transformation, spatially resolved measurements are obtained.

The spectroscopy methods divide into two groups: The measurement of $n_{e}$ by Balmer line ratios and by the rotational temperature of molecules is only suitable for ionizing plasmas; the measurement of $n_{e}$ by the Stark broadening of Paschen lines and of $T_{e}$ by Paschen line ratios is only applicable for recombining plasmas.

For the evaluation of these methods, different plasma conditions are produced in PSI-2. The plasma generator is capable of producing deuterium plasmas with electron densities of up to $10^{13} \mathrm{~cm}^{-3}$ and electron temperatures of up to $20 \mathrm{eV}$. Additional measurements with a Langmuir double probe are conducted for comparison with the spectroscopy measurements.

A collisional-radiative model in the Yacora code is used to compare measured Balmer line emissions with the calculation and to investigate which reaction channels influence the recombination in PSI-2.

\section{INTRODUCTION}

Linear plasma devices can contribute to the specific open issues on the road to the fusion reactor, particularly in the field of plasma-material interaction (PMI) studies. ${ }^{1}$ The JULE-PSI project foresees a linear plasma generator in a hot cell environment for PMI studies with neutron activated and toxic targets. ${ }^{2}$ In this environment the complexity of a diagnostic system must be low enough to keep the necessary maintenance and possible technical failures to a minimum. The optical emission spectroscopy does not require any moving/electrical parts inside the hot cell. Therefore, spectroscopy is an attractive option to measure the plasma parameters in JULE-PSI.

The ITER divertor will be operated in a partially detached regime, with plasma recombination occurring in significant amounts ${ }^{3}$. The development of techniques is necessary to determine parameters of recombining plasma.

In this study, the applicability of passive deuterium spectroscopy has been tested in both ionizing and recombining regimes at the PSI-2 linear plasma generator, which serves as a pilot experiment for JULE-PSI.

\section{EXPERIMENTAL SETUP}

\section{II.A. PSI-2 linear plasma generator}

The PSI-2 linear plasma generator (Fig. 1) produces a cylindrical plasma column with a length of $2.5 \mathrm{~m}$ and a diameter of $6 \mathrm{~cm}$ in the target region. In this region material samples can be moved inside the plasma by a target manipulator for PMI experiments. The targets as well as the plasma can be observed through various ports in three segments in the target exposure chamber with a distance of $1.5,1.85$ and $2.2 \mathrm{~m}$ from the plasma source.

The plasma is produced in an arc-discharge plasma source with heated $\mathrm{LaB}_{6}$ cathode. The plasma source is currently capable of producing deuterium plasmas which reach electron densities up to $10^{13} \mathrm{~cm}^{-3}$, electron temperatures of up to $20 \mathrm{eV}$, and a parallel ion flux of up to $10^{19} \mathrm{~cm}^{-2} \mathrm{~s}^{-1}$ in the target region. The profile of the plasma column is influenced by the geometry of the cylindrical cathode: The plasma column is hollow with a maximum of electron temperature and density at $\mathrm{r}=2.5 \mathrm{~cm}$ in the target region.

When the plasma drifts out of the plasma source, it is confined by a magnetic field of $100 \mathrm{mT}$ on the axis in the target region. The end of the plasma column is defined by a cooled neutralizer plate made of copper. 


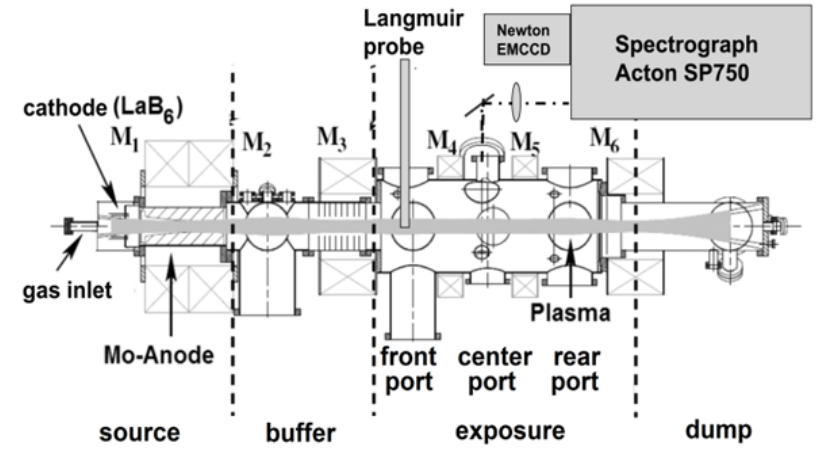

Fig. 1. PSI-2 experimental setup. The main parts of the device and of the spectroscopic system are indicated. M1 - M6 are the magnetic field coils.

In this paper, two cases are investigated: first, ionizing plasma with a variation in discharge power; second, recombining plasma with a constant discharge power. Recombination is realized by variation of the neutral gas pressure in the target area by additional gas inlets. It starts at a certain neutral gas pressure $(\sim 0.004$ mbar $)$ and is visible as a blue light emission inside the pink plasma column. Due to the hollow temperature and density profiles, recombination starts in the center of the plasma, where $T_{e}$ is lower than in the edge.

\section{II.B. Plasma diagnostics}

Measurements were performed by a 2D imaging spectrometer (Acton SP750 $75 \mathrm{~cm}$ spectrograph combined with an Andor Newton EMCCD camera) and a movable Langmuir double probe. With the $2 \mathrm{D}$ spectrometer a radial profile of each spectral line along the diameter of the plasma column can be obtained. As an example, figure 2 shows a spectrum of the recombining plasma.

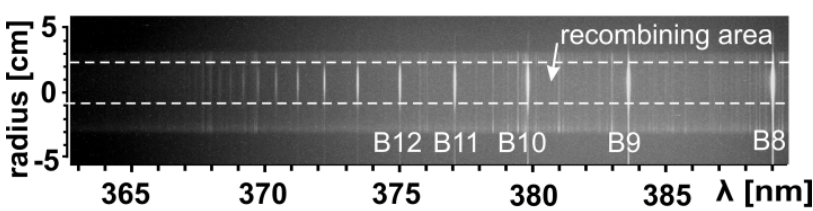

Fig. 2. Example image of the EMCCD camera for Balmer series lines. The recombining area within the plasma column is indicated.

In this image the radial profile of the plasma column is visible, as well as high-n Balmer lines, which mostly radiate only in the recombining area. Each point of this profile is a line integrated measurement. The profiles can be converted with an inverse Abel transformation to obtain the spatially resolved light emission along the plasma radius. This conversion is only possible when the profiles are rotationally symmetric, which is not the case for the Balmer lines in figure 2. However, the inverse Abel transformation of a rotationally symmetric line profile shows that the actual light emission is mainly located in the edge of the plasma, corresponding to the hollow plasma profile.

A Langmuir double probe was used to compare $n_{e^{-}}$ and $\mathrm{T}_{\mathrm{e}}$-measurements. The probe can be moved to measure the radial profile of the plasma.

Measurements with the spectrometer were done in the target area at the center segment (as shown in figure 1) and at the front segment, where the Langmuir probe is located.

\section{METHODS AND RESULTS}

\section{III.A. Electron density: Balmer line ratios}

The intensity ratio of $\mathrm{D}_{\alpha}$ and $\mathrm{D}_{\gamma}$ lines is a function of electron density. In this experiment, $\mathrm{D}_{2}$ plasma with variation in discharge power was produced. The ADAS database was used to deduce the electron density from the Balmer line ratios. ${ }^{4}$ In figure 3 the profiles of $n_{e}$ measured by spectroscopy are compared with the Langmuir probe results.

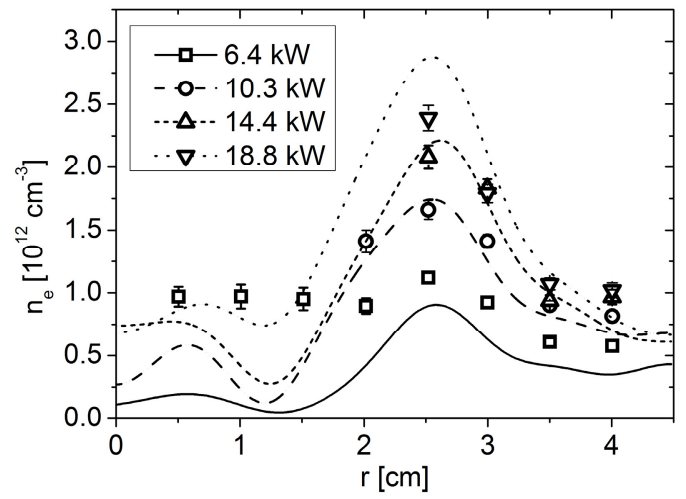

Fig. 3. Radial profile of $\mathrm{n}_{\mathrm{e}}$ by $\mathrm{D}_{\alpha} / \mathrm{D}_{\gamma}$ (lines) and by the Langmuir probe (symbols) under variation of the discharge power as noted in the legend. The error bars are the standard deviation of averaged measurements.

As expected, the maximal electron density is found at $\mathrm{r}=2.5 \mathrm{~cm}$, and the density increases with increasing discharge power. The Langmuir probe values confirm the results in the plasma edge, but a deviation of the results in the plasma center is observed. This can be explained by an increasing error of the line intensity data in the center due to the inverse Abel transformation, or by an influence of the Langmuir probe on the plasma parameters. The probe data in the center are only available for the lowest discharge power because of overheating of the probe for a higher discharge power. 


\section{III.B. Electron density: Fulcher band lines}

The following method was previously investigated at the TEXTOR tokamak. ${ }^{5}$ It was shown that the rotational temperature $\mathrm{T}_{\text {rot }}$ of $\mathrm{D}_{2}$ excited molecules depends linearly on the electron density: ${ }^{6}$

$$
\begin{gathered}
T_{r o t}[\mathrm{~K}]=3.0( \pm 0.4) \cdot 10^{-11} n_{e}\left[\mathrm{~cm}^{-3}\right] \\
+0.5 \cdot T_{\text {gas }}[\mathrm{K}]+137
\end{gathered}
$$

where $\mathrm{T}_{\text {gas }}$ is the gas temperature.

The rotational temperature is obtained by a Boltzmann plot of the Fulcher $\mathrm{Q}(0-0)$ band emission lines. The corresponding transition $\left(3 p^{3} \Pi_{u} \rightarrow 2 s^{3} \Sigma_{g}^{+}\right)$of the Fulcher lines is sensitive to the electron density in the range of $\mathrm{n}_{\mathrm{e}}=10^{12}-10^{15} \mathrm{~cm}^{-3}$ (Ref. 5).

The linear dependence obtained for TEXTOR is compared to the relation between rotational temperature and $n_{e}$ (measured with the Langmuir probe) in PSI-2 in figure 4. Here, the data from the same experiment as described in the previous section is used. The plot shows that the PSI-2 data follow the linear dependence found in TEXTOR with constant gas temperature for densities higher than $\approx 10^{12} \mathrm{~cm}^{-3}$. The deviation at lower densities can be explained by a gas temperature change in PSI-2, which also influences the rotational temperature according to equation (1).

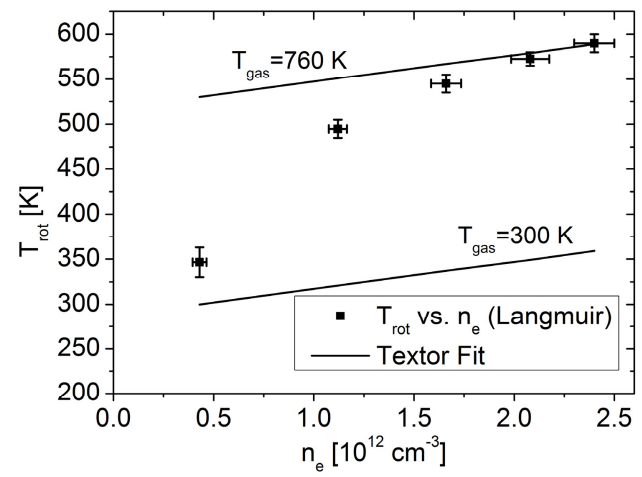

Fig. 4. Rotational temperature as a function of electron density. Measurements by the Langmuir probe (symbols) are compared to the relation (Eq. 1) obtained for TEXTOR (lines). The error bars are the standard deviation of averaged measurements.

\section{III.C. Electron density: Paschen line Stark broadening}

While the methods described in Sec. III.A and III.B are only applicable to ionizing plasma, the following techniques are suitable for recombining plasma. Such a plasma was produced as described in section II.A, and the emission from the recombining region was observed with the imaging spectrometer at the front $(\mathrm{z}=1.5 \mathrm{~m})$ and middle $(\mathrm{z}=1.85 \mathrm{~m})$ segments of the target exposure region. As $T_{e}$ is very low (below $1 \mathrm{eV}$ ) in recombining plasmas, the Langmuir probe provides no reliable results and cannot be used for comparison.

For the determination of electron density in the recombining region, the Stark broadening of the Paschen line $n=10 \rightarrow 3$ is used. $^{7}$

$$
\Delta \lambda[\mathrm{nm}]=6 \cdot 10^{-7} n^{\frac{2}{3}} \cdot \lambda^{2} \cdot u^{2} \cdot \frac{1}{Z}
$$

Here, $n\left[\mathrm{~cm}^{-3}\right]$ is the electron density, $\lambda[\mathrm{nm}]$ the wavelength, $u$ the quantum number of the Paschen line, and $Z$ the atomic number of the element.

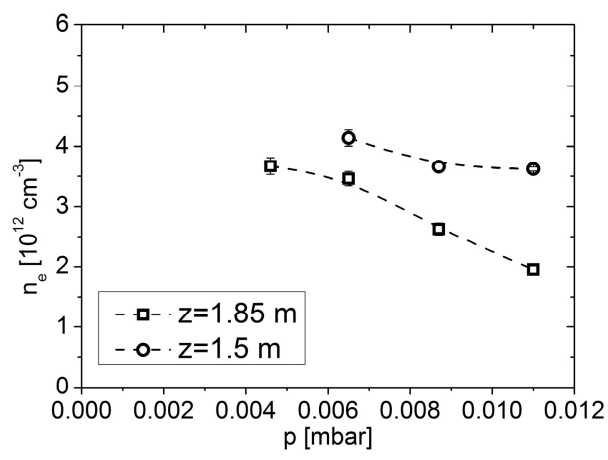

Fig. 5. Electron density as a function of the neutral gas pressures at two positions along the z-axis in recombining plasma. The error bars are the uncertainty of line profile fit.

Figure 5 shows that electron density decreases with increasing neutral gas pressure due to recombination. Also, electron density is higher in the z-position closer to the source than in the z-position further away for the same neutral gas pressure. It confirms the visual observation of a better pronounced recombination zone further away from the source.

\section{III.D. Electron temperature: Paschen lines ratio}

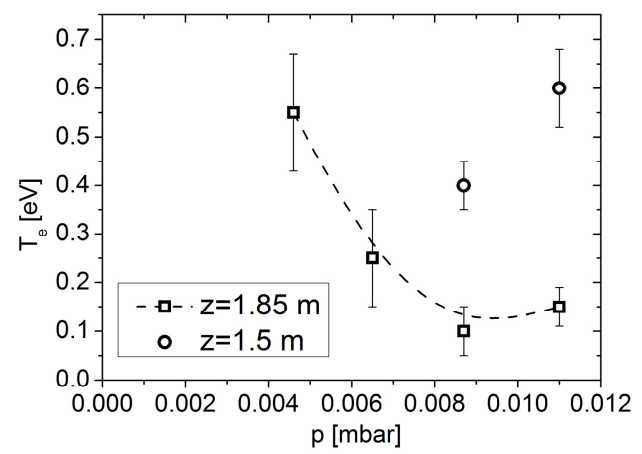

Fig. 6. Electron temperature as a function of neutral gas pressure at two positions along the $\mathrm{z}$-axis in recombining plasma. The error bars are the standard deviation of averaging over whole recombining area. 
For the same experiment as in Sec. III.C, the electron temperature in the recombining region was determined by the intensity ratio of Paschen $n=9 \rightarrow 3$ and $n=7 \rightarrow 3$ lines. The relation between line ratio and electron temperature was again obtained from ADAS data. ${ }^{4}$

Figure 6 shows that electron temperature also decreases with increasing recombination, and that it is higher at the position closer to the plasma source. Because of a low light intensity for the measurements at $\mathrm{z}=1.5 \mathrm{~m}$, only the two datapoints with the highest neutral gas pressure could be evaluated.

\section{SIMULATION OF BALMER LINE EMISSIONS}

Calculations with a collisional radiative $\operatorname{model}^{8}$ for low temperature, low density plasmas (based on the Yacora code) were performed and compared with experimental data. The model calculates the emission of Balmer lines for a given set of input parameters, particularly electron density, electron temperature and the density of different hydrogen ions and molecules. The effect of the $\mathrm{D}^{+}$and $\mathrm{D}_{2}^{+}$density on the results can be taken as an evidence on whether molecular activated recombination (MAR, $\mathrm{D}_{2}^{+}$) or electron ion recombination $\left(\mathrm{EIR}, \mathrm{D}^{+}\right)$has a bigger influence under the particular plasma conditions in PSI-2. Rate coefficients suggest that both processes could have an influence in recombining plasma with $\mathrm{T}_{\mathrm{e}}<1 \mathrm{eV}$.

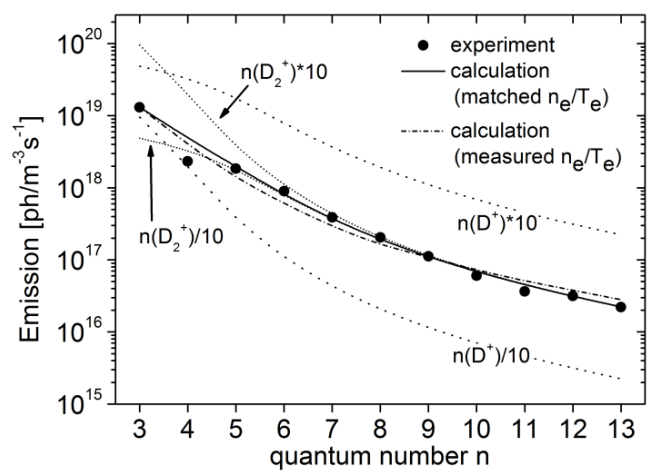

Fig. 7. Emission of Balmer lines in a recombining plasma by measurements and by Yacora simulation

Figure 7 shows an example for the recombining plasma described in section II.A at a neutral gas pressure of 0.004 mbar. The experimentally obtained values are drawn as single points, the calculation with the measured $\mathrm{n}_{\mathrm{e}}$ and $\mathrm{T}_{\mathrm{e}}$ values $\left(\mathrm{n}_{\mathrm{e}}=4 \cdot 10^{12} \mathrm{~cm}^{-3}, \mathrm{~T}_{\mathrm{e}}=0.5 \mathrm{eV}\right)$ as a dasheddotted line, and the calculation with $n_{e}$ and $T_{e}$ values as free parameters as a solid line. The result of the bestmatch of $\mathrm{n}_{\mathrm{e}}$ and $\mathrm{T}_{\mathrm{e}}$ is $\mathrm{n}_{\mathrm{e}}=5.4 \cdot 10^{12} \mathrm{~cm}^{-3}$ and $\mathrm{T}_{\mathrm{e}}=0.22 \mathrm{eV}$, fairly close to the measured values.

The dotted lines show the impact of a numerical variation of the $\mathrm{D}^{+}$or $\mathrm{D}_{2}^{+}$density by a factor of 0.1 and 10 . Though the variation of $\mathrm{D}^{+}$has a bigger effect than the variation of $\mathrm{D}_{2}^{+}$, both corresponding processes (EIR and MAR) seem to be important for the plasma conditions in this particular experiment. However, the numerical matching of the fraction of $\mathrm{D}_{2}^{+}$and $\mathrm{D}^{+}$of the total ion amount provides rather low values: $3.5 \%$ for $\mathrm{D}^{+}$and $0.001 \%$ for $\mathrm{D}_{2}^{+}$; suggesting that $\mathrm{D}_{3}^{+}$is the dominant species in the plasma. This result has still to be confirmed by further simulations and measurements.

\section{SUMMARY}

Several spectroscopic methods were applied for the determination of electron density and electron temperature for ionizing and recombining deuterium plasmas in the PSI-2 linear plasma generator. The spectroscopic measurements are performed with an imaging spectrometer, which can produce spatially resolved radial profiles of the cylindrical plasma column. The results are compared to Langmuir probe measurements, where available. The applied methods deliver reasonable results, but some limitations, like on a certain temperature- or density range, have to be taken in account. A simulation with a collisional radiative code indicates an influence on the recombination of both electron ion recombination (EIR) and molecular activated recombination (MAR).

\section{REFERENCES}

1. A. KRETER, Transactions Fusion Sci. Technol., 59, 51 (2011).

2. B. UNTERBERG et al., Fus. Eng. Des., 86, 1797 (2011).

3. Progress in the ITER Physics Basis, Nucl. Fusion, 47, (2007).

4. H. P. SUMMERS, "The ADAS User Manual, version 2.6, http://www.adas.ac.uk" (2004).

5. S. BREZINSEK, ,Untersuchung von atomarem und molekularem Wasserstoff vor einer Graphitoberfläche in einem Hochtemperatur-Randschichtplasma“, FZ-Jülich Report, Jül-3962 (2002)

6. G. SERGIENKO et al., „Molecular Deuterium Behaviour in Tungsten Divertor on JET", submitted to Journal of Nuclear Materials

7. V. A. SOUKHANOVSKII et al., "Electron density measurements in the National Spherical Torus Experiment detached divertor region using Stark broadening of deuterium infrared Paschen emission lines", Rev. Sci. Instrum., 77, 10F127 (2006)

8. D. WÜNDERLICH, S. DIETRICH and U. FANTZ, Journal of Quantitative Spectroscopy \& Radiative Transfer, 110, 62-71 (2009)

9. OHNO et al., "Experimental Evidence of Molecular Activated Recombination in Detached Recombining Plasmas", Phys. Rev. Lett., 81, 818-821 (1998) 